\title{
INNOVACIÓN DOCENTE EN LOS GRADOS DE EDUCACIÓN INFANTIL Y PRIMARIA. DANDO SIGNIFICADO Y CONTEXTO A LOS APRENDIZAJES DE AULA EN EL ESPACIO URBANO ${ }^{1}$
}

\author{
TEACHING INNOVATION IN EARLY YEARS AND PRIMARY EDUCATION TEACHER \\ TRAINING DEGREES. GIVING MEANING AND CONTEXT TO CLASSROOM LEARNING IN \\ THE URBAN ENVIRONMENT
}

\author{
María García-Cano Torrico \\ maria.garciacano@uco.es \\ Eva Hinojosa Pareja \\ ehinojosa@uco.es \\ Elba Gutiérrez-Santiuste \\ egsauntiuste@uco.es \\ $\mathbf{M}^{\mathrm{a}}$ José Martínez Carmona \\ mjmartinez@uco.es \\ Universidad de Córdoba
}

\begin{abstract}
This paper describes an innovation project developed during the 2015/16 academic year within the context of the subjects Coexistence within the School and Culture of Peace studied as part of the Early Years and Primary Education Teacher Training Degree Courses taught at the University of Cordoba. The aim has been to put students in contact with social and cultural organisations, associations and collectives in the city so that they are able to incorporate into the classroom critical analysis of the most relevant issues in today's society. This project is also working to provide an instrument for teaching reflection. The utility of this project includes: improving student learning, transcending the mere transmission of knowledge in the classroom and moving towards the generation of learning experiences outside the classroom as well; and increasing coordination between university faculty members as a group and also between these faculty members and social agents in the city.
\end{abstract}

Keywords: pre-service teacher education; social groups; social engagement; teacher coordination; education.

\section{Resumen}

El artículo describe el proyecto de innovación docente desarrollado durante el curso 2015/16 en el contexto de las asignaturas de Convivencia en la Escuela y Cultura de Paz de los estudios de Grado de Educación Infantil y Educación Primaria de la Universidad de Córdoba. El objetivo que ha pretendido ha sido poner en relación al alumnado con organizaciones, asociaciones y colectivos del ámbito social y cultural en la ciudad con el propósito de incorporar a las aulas el análisis crítico de las cuestiones sociales más relevantes de la sociedad actual. Este proyecto, además, ha pretendido ser un instrumento para la reflexión docente. Entre las utilidades del proyecto señalamos: la mejora de los aprendizajes del alumnado, trascendiendo de la mera transmisión de saberes en el aula hacia la producción de experiencias de aprendizaje también fuera de ella; en segundo lugar, servir de impulso para la coordinación entre el profesorado universitario y de éste con los agentes sociales de la ciudad.

Palabras clave: educación, coordinación docente, formación docente; organizaciones sociales; participación social.

\section{INTRODUCCIÓN}

El inicio de los nuevos Grados de Educación Infantil y Primaria encargados de la formación académica de maestros y maestras ha permitido enfatizar una formación basada en competencias orientada a la construcción de saberes conectados con el tejido social (Ministerio de Educación 2010; CRUE, 2015). Se pretende que la formación académica revierta de alguna manera en mejoras tanto desde el punto de vista de oportunidades para la inserción en el mercado de trabajo, como también que impacte y favorezca en el ámbito social y cultural. Se trata de una apuesta por la responsabilidad social de la institución universitaria, una visión concreta de la transferencia que subraya el valor del conocimiento en función de sus contribuciones para el bien común.

Para llevar a cabo dicha formación, en los últimos años se ha abierto una importante línea de trabajo sobre la significación de evidencias científicas que muestren cuáles son las prácticas más exitosas o de alto impacto en el aprendizaje del alumnado universitario en general y en la formación inicial del profesorado de Infantil y Primaria en particular (Kilgo, Ezell-Sheets y Pascarella, 2015). Al respecto, se reclama la importancia de acercar a los estudiantes a investigaciones y trabajos científicos que les permitan el debate y la toma de postura (Wineburg, 2006), la puesta en práctica de procedimientos de trabajo intelectual (revisión por pares, grupo de lectura y escritura, estudio de caso,

1 El proyecto subvencionado tenía por título: De la transmisión de contenidos a la arquitectura de experiencias. Dando contexto y significado al aprendizaje del aula en el espacio urbano (II) (ref. : 2015-2-6006) 
entre otros) emulando a la comunidad científica por parte de estudiantes y profesorado (Flecha, Racionero, Tintoré y Arbós, 2014) o la realización de trabajos que exijan el compromiso cívico y la responsabilidad ciudadana con el objeto de aplicar conocimientos y habilidades en la solución de problemáticas fuera del aula (Kuh, 2008).

De forma particular y a pesar de la relevancia que se le ha ido concediendo a la adquisición de competencias ciudadanas y al compromiso cívico durante los últimos años desde las declaraciones y los discursos -de forma particular en la formación inicial del profesorado a la capacidad reflexiva, la de pensar críticamente o la de realizar análisis de las cuestiones más relevantes del contexto social-, aun encontramos un importante camino por recorrer en el ámbito de la práctica diaria de la docencia universitaria en las asignaturas de Grado.

El proyecto de innovación propuesto se asienta sobre estos supuestos en tanto que ha brindado la oportunidad al alumnado de establecer relaciones con organizaciones, asociaciones y colectivos del ámbito social y cultural de carácter no gubernamental (en adelante colectivos colaboradores) en la ciudad promoviendo, de este modo, el intercambio de conocimientos desde los que interpretar el contenido docente trabajado en el programa de la asignatura. Asunto que se hace especialmente apreciable dado el papel que juegan los movimientos sociales en la configuración social del espacio público, así como su protagonismo en la demanda de transformaciones sociales, culturales y políticas más amplias (Castells, 2012).

Conectar los aprendizajes adquiridos en la universidad con las acciones, necesidades, demandas de distintos grupos sociales posibilita el desarrollo de habilidades y competencias para el aprendizaje académico y la competencia social ý cívica. Esta asunción se asienta en distintas investigaciones que de forma sólida fundamentan nuestro trabajo de innovación docente que describimos en este artículo.

En primer lugar, la literatura acentúa la importancia de generar metodologías que permitan otorgar un "papel activo del estudiante" como constructor del conocimiento (Pujolás, 2007; Pujolás, 2008). En paralelo, este nuevo protagonismo del estudiante implica un nuevo rol del papel del profesorado pasando de ser el centro del proceso de enseñanza-aprendizaje a tener una función de mediador, facilitador, arquitecto o incluso de co-investigador del mismo (Brubaker, 2012).

En segundo lugar, referencias teóricas proponen metodologías que permitan desafiar intelectualmente (Bain, 2007) a los y las estudiantes partiendo de motivaciones intrínsecas. En tales casos se entiende por aprendizaje un proceso de renovación en el que el alumnado da un salto más allá de ellos mismos y de los límites que están marcados (Atkinson, 2011).

En tercer lugar, una línea de trabajo fructífera insiste en la importancia de caminar hacia el diseño y desarrollo de un currículum global en la formación inicial del profesorado. Nuestros futuros docentes van a desarrollar su labor profesional en una sociedad cada vez más interconectada e interdependiente. Aprender a enseñar en contextos híbridos, cambiantes y globales requiere una enseñanza universitaria que trascienda las tradicionales disciplinas e incorpore marcos de conocimiento más generales que atiendan a diferentes dimensiones del ser humano (Reynolds et al., 2015). En coherencia con este planteamiento, el contenido teórico y práctico de la asignatura desarrolla temáticas vinculadas a los factores globales que condicionan la convivencia, como la sostenibilidad, ciudadanía, Derechos Humanos y cultura de paz, desigualdades estructurales y sociales, diversidad cultural y de género, etc. Todos ellos, tópicos esenciales desde el punto de vista de la Educación Global (Ferguson, Macqueen y Reynolds, 2014).

\section{OBJETIVOS}

Los objetivos principales han sido:

a. Promover en el aula universitaria el intercambio de conocimientos a través del contacto con organizaciones y colectivos sociales que revierta en una mejora docente.

b. Reflexionar sobre el proceso de innovación docente emprendido y analizar los logros, obstáculos y desafíos que acontezcan en el transcurso del proyecto.

Para ello hemos establecido una serie de objetivos operativos en los que hemos concretado nuestras actuaciones:

I. Identificar colectivos colaboradores que permitan al alumnado significar los contenidos abordados en la asignatura Convivencia en la escuela y Cultura de Paz.

II. Facilitar el acceso del alumnado a espacios de colaboración colectiva, cercanos y experienciales que fomenten su sensibilidad social, la solidaridad y el compromiso social.

III. Indagar a través de documentos, entrevistas y observaciones en el trabajo desarrollado por dichos colectivos para conocer los objetivos, demandas, problemáticas y situaciones concretas en las que trabajan.

IV. Revisar las guías de trabajo elaboradas para orientar al alumnado en la metodología a seguir para la realización de las prácticas propuestas y en el establecimiento de vínculos conceptuales y reflexivos entre la experiencia y los contenidos expuestos en el programa.

V. Identificar, desde el punto de vista docente, los factores que facilitan y/o dificultan el proceso de enseñanzaaprendizaje, así como los logros y los obstáculos alcanzados con esta metodología en el desarrollo de la asignatura.

VI. Fomentar el conocimiento activo, comprometido y crítico del alumnado universitario con el contexto y sus condicionantes. 
VII. Consolidar una red de trabajo conjunto e intercambio entre colectivos sociales y universidad.

\section{DESCRIPCIÓN}

La experiencia de innovación docente consta de varias fases:

Fase 1. Conocimiento previo y diseño del trabajo. Los fines de esta fase consisten en facilitar el acceso al alumnado a espacios de colaboración colectiva, cercanos y experienciales que fomenten su sensibilidad social, la solidaridad y el compromiso social. También es necesario identificar los colectivos, asociaciones y organizaciones no gubernamentales que permitan al alumnado significar los contenidos abordados en la asignatura e identificar, desde el punto de vista docente, los factores que facilitan y/o dificultan el proceso de enseñanza-aprendizaje, así como los logros y los obstáculos alcanzados con esta metodología.

Fase 2. Indagación dentro y fuera del aula. Los fines relacionados consisten en la indagación por parte del alumnado sobre la entidad colaboradora para conocer los objetivos, demandas, problemáticas y situaciones concretas en las que trabajan. Por otro lado, deben establecer los vínculos conceptuales y reflexivos entre la experiencia y los contenidos expuestos en el programa.

Fase 3. Esta fase consiste en compartir saberes, transferir experiencias y construir nuevo conocimiento. Es fundamental en esta fase la conexión del conocimiento generado por el alumnado a partir de su experiencia de prácticas con el contenido impartido a lo largo de la asignatura. El fin es fomentar el conocimiento activo, comprometido y crítico del alumnado universitario con el contexto y sus condicionantes.

Fase 4. Evaluación y seguimiento del proyecto. Los fines son identificar, desde el punto de vista docente, los factores que facilitan y/o dificultan el proceso de enseñanza-aprendizaje, así como los logros y los obstáculos alcanzados con esta metodología en el desarrollo de la asignatura. Igualmente se pretende consolidar una red de trabajo conjunto e intercambio entre colectivos colaboradores y universidad ya iniciada en cursos previos que ve incrementada su demanda desde ambos extremos.

\section{Metodología}

Diferenciamos entre la metodología desplegada para desarrollar la experiencia docente y la metodología seguida para proceder al análisis de dicho proceso.

\subsection{Metodología de LA EXPERIENCIA DOCENTE}

La metodología docente se ajusta a las diferentes fases descritas en el apartado Descripción.

En la primera fase el alumnado se distribuye en grupos de trabajo de entre cinco/seis componentes, para poder llevar a cabo las distintas tareas que la asignatura requiere. El profesorado identifica la organizaciones colaboradoras que permitan al alumnado significar los contenidos abordados en la asignatura.

Tras estas acciones procedemos a explicar el trabajo a realizar por parte del alumnado y facilitamos un listado de organizaciones sociales susceptibles de contacto de acuerdo a distintos ámbitos de acción, como son: salud; exclusión social, grupos marginados; discapacidad, dependencia; promoción y defensa de Derechos Humanos; defensa de los derechos de los animales, cooperación al desarrollo; ocio, cultura, deporte; educación. Este listado es revisado y actualizado cada curso académico. Para las actuaciones de contacto y establecimiento de relaciones con el tejido asociativo de la ciudad se han establecido reuniones particulares y asistencia a las asambleas de Plataforma Córdoba Solidaria.

A partir de esta información, cada grupo de alumnos-as selecciona una entidad colaboradora con la que realizará las siguientes fases de trabajo y con la que mantendrá un primer contacto solicitando colaboración. En este punto, se hace imprescindible conocer, desde la perspectiva del docente, los factores que facilitan y/o dificultan el proceso de enseñanza-aprendizaje.

En una segunda fase el alumnado analiza documentos facilitados por cada una de las organizaciones sociales, realiza entrevistas y lleva a cabo observaciones directas del trabajo desarrollado por dichos colectivos. Los profesoras en esta fase revisan las guías de trabajo elaboradas para orientar al alumnado en la metodología a seguir en la realización de las prácticas propuestas. Se establece un calendario y plazos para cada una de las tareas a realizar por el alumnado y se realiza un seguimiento detallado de cada una de las actuaciones de los grupos reducidos de trabajo.

Durante esta segunda fase, se desarrollan en el aula talleres/ seminarios por parte de las entidades colaboradoras invitadas por las profesoras o a través de los grupos de alumnas-os.

Durante el curso 2015-16 se han impartido 6 talleres. Concretamente los colectivos invitados han intervenido en el aula desde los siguientes ámbitos de trabajo: Exclusión social, grupos marginados (Mujeres en Zona de Conflicto, Estrella Azahara), Cooperación al desarrollo (Paz con Dignidad, Ingeniería Sin Fronteras), Dependencia y Discapacidad (Red Española de Madres y Padres Solidarios con las enfermedades raras), Educación (Asociación de Educación Ambiental y El bosque animado).

También se han realizado talleres metodológicos por parte de las profesoras en relación a: qué es y qué habilidades se requieren para la fase del trabajo de campo, realización de entrevistas, observaciones, proceder 
posteriormente al análisis documental y cómo realizar un informe de investigación. Anteriormente las profesoras elaboraron un dossier de material de carácter metodológico, así como guías de trabajo que permitan al alumnado llevar a cabo el trabajo de campo siguiendo criterios metodológicos necesarios y acordes con el nivel de conocimientos del alumnado.

Esta fase incluye también, en primer lugar, la redacción de los ensayos reflexivos en grupos de trabajo sobre la información obtenida y su relación con los aspectos teóricos tratados en la asignatura y, en segundo lugar, la realización de recursos didácticos y documentos explicativos de los mismos que sean útiles para la entidad colaboradora.

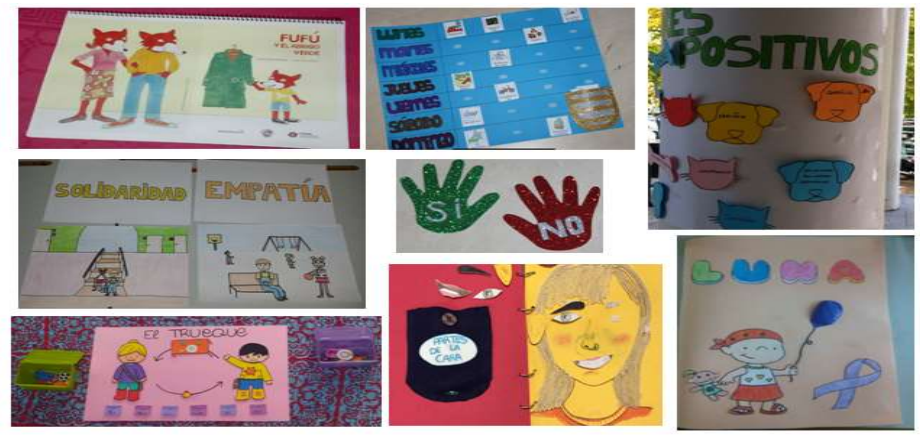

Figura 1: Ejemplo de recursos didácticos realizados por el alumnado

Por parte de las profesoras se realizó un cuaderno de clase donde se han anotado los aspectos más destacados sobre la dinámica seguida y el proceso de tutorización.

En la tercera fase se realiza la exposición conjunta de los trabajos realizados a través de una "feria" abierta a toda la comunidad educativa de la Facultad de Ciencias de la Educación en el hall y los pasillos de entrada a la misma. Debido al alto número de alumnado se realizan dos turnos para la feria: un turno de mañana y uno de tarde donde, asimismo, las organizaciones colaboradoras, si lo desean, asisten y forman parte de este evento.

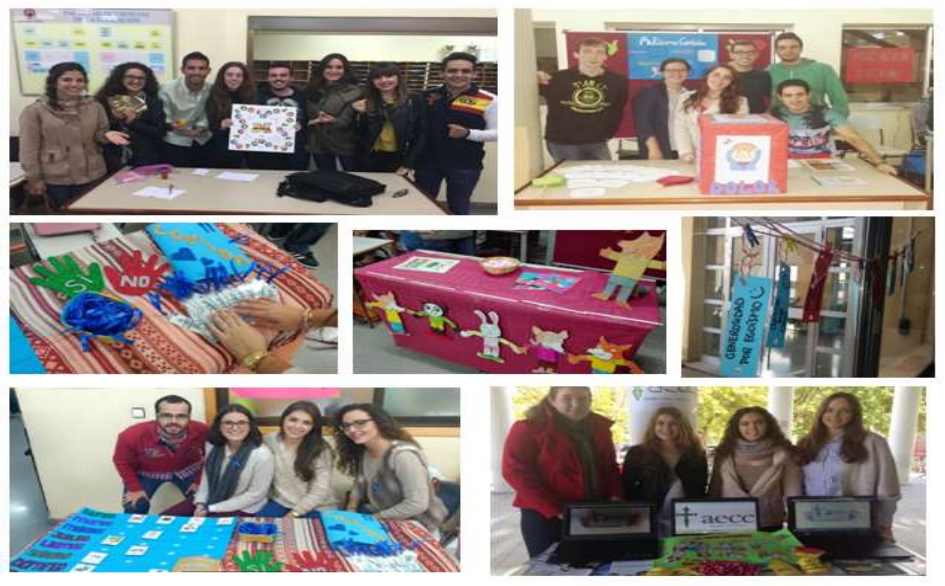

Imagen 2: Ejemplo de stands de grupos de trabajo en la feria

La experiencia docente finaliza con la evaluación y seguimiento del proyecto (fase 4). La metodología utilizada en esta fase consiste en la celebración de reuniones de trabajo con otras posibles organizaciones colaboradoras de la ciudad para presentar el proyecto y establecer mecanismos de participación. Por otro lado, para el alumnado resulta de utilidad la elaboración de rúbricas tanto de autoevaluación como para la evaluación de los grupos entre ellos.

\subsection{METODOLOGÍA DE ANÁLISIS DE LA EXPERIENCIA DOCENTE}

El número de alumnado matriculado en el curso 2015/16 en Convivencia en la Escuela y Cultura de Paz de los estudios de Grado de Educación Infantil asciende a 186 y de Grado de Educación Primaria a 253.

Con respecto al análisis del impacto del proyecto en el alumnado aplicamos un cuestionario inicial con el objetivo de conocer los conocimientos previos sobre organizaciones y colectivos sociales y su experiencia previa en metodologías de enseñanza-aprendizaje relacionadas con este proyecto. En segundo lugar, realizamos grupos focales para profundizar en las expectativas, conocimientos previos y experiencias del alumnado. Con el objeto de valorar la experiencia desarrollada, problemáticas surgidas y potencialidades identificadas por el alumnado utilizamos un cuestionario final y grupos focales terminada la asignatura.

Para las actividades de reflexión sobre la actuación docente del profesorado se utiliza una metodología cualitativa, de carácter interpretativo, mediante el análisis de contenido del diario de campo y los grupos focales entre las 
profesoras, realizados cl inicio y final de curso. El objetivo fundamental ha sido valorar los aprendizajes y dificultades en el proceso de enseñanza-aprendizaje.

\section{RESULTADOS OBTENIDOS}

Estructuramos los resultados alcanzados en relación a los distintos actores que han conformado parte de su desarrollo: alumnado, profesorado y organizaciones sociales.

Con respecto al alumnado de los Grados de Infantil y Primaria, relacionamos los resultados obtenidos teniendo como referente las competencias de cada Grado señaladas para cada una de las asignaturas (ver Tabla 1):

\begin{tabular}{|l|l|}
\hline Tabla 1. & \multicolumn{1}{|c|}{ Contenido } \\
\hline Competencia & $\begin{array}{l}\text { Analizar e incorporar de forma crítica las cuestiones } \\
\text { más relevantes de la sociedad actual que afectan a la } \\
\text { educación familiar y escolar; cambios en las relaciones } \\
\text { de género e intergeneracionales; multiculturalidad e } \\
\text { interculturalidad; discriminación e inclusión social y } \\
\text { desarrollo sostenible. }\end{array}$ \\
\hline CM2.5. & $\begin{array}{l}\text { Conocimiento de los procesos de interacción y } \\
\text { comunicación en el aula y mostrar habilidades para } \\
\text { abordarlos. }\end{array}$ \\
\hline CM2.6. & $\begin{array}{l}\text { Abordaje y resolución de problemas de disciplina y } \\
\text { situaciones de convivencia desde la perspectiva de la } \\
\text { educación para la paz. }\end{array}$ \\
\hline CM2.7. & $\begin{array}{l}\text { Promoción del trabajo cooperativo y el trabajo y } \\
\text { esfuerzo individuales. }\end{array}$ \\
\hline CM2.8. & $\begin{array}{l}\text { Promoción de acciones de educación en valores } \\
\text { orientadas a la preparación de una ciudadanía activa y } \\
\text { democrática en el marco de los derechos humanos. }\end{array}$ \\
\hline CM2.11. & $\begin{array}{l}\text { Conocimiento y aprendizaje de experiencias } \\
\text { innovadoras en educación Primaria-Infantil. }\end{array}$ \\
\hline
\end{tabular}

A propósito de la competencia CM3.4. su adquisición ha sido posible a través del trabajo consistente en el mapeo, contacto, realización de entrevistas, observaciones, análisis documental y su análisis reflexivo en el contexto de la asignatura (con nodos teóricos como Mundo global, Ciudadanía, Ética del Cuidado, Diversidad y Desigualdad). El alumnado ha realizado dos trabajos escritos independientes: ensayos reflexivos y documentos explicativos de los recursos didácticos realizados. En ambos se refleja el análisis del contexto social más amplio desde una perspectiva crítica y atendiendo a distintas visiones y perspectivas.

A propósito de la competencia CM2.5 el alumnado ha descrito proyectos de educación desarrollados por distintas organizaciones, en entornos formales o informales, de una gran diversidad y complejidad que le permite tener claves suficientes para analizar y conocer procesos de interacción y comunicación. Asimismo, la estrategia más utilizada en el desarrollo de la innovación ha sido el diálogo a través de las entrevistas.

A propósito de la competencia CM2.6. A través de la descripción y el análisis de las necesidades y demandas de los colectivos, el alumnado ha diseñado actuaciones y recursos (cuentos, juegos didácticos, vídeos,...) dirigidas hacia la promoción de la cultura de paz y la no violencia. Estos recursos han sido expuestos para darlos a conocer tanto a las organizaciones como a la comunidad universitaria de la Facultad de Ciencias de la Educación.

A propósito de la competencia CM2.7, los trabajos desarrollados durante todo el curso han sido realizados en grupos de 5-6 personas y han requerido de una alta dosis de planificación y coordinación tanto para los primeros contactos, como para la realización de entrevistas y observaciones, redacción del informe final, elaboración de recursos y devolución de resultados. La coordinación y relevancia del trabajo cooperativo desarrollado se ha visto reflejada en el apartado de Metodología redactado por el alumnado en los ensayos entregados a las profesoras, donde se describe el procedimiento seguido para la elaboración de los trabajos.

A propósito de la competencia CM2.8, el alumnado ha diseñado recursos y actuaciones útiles para la promoción de la participación en los contextos social y cultural de la ciudad. Muchos de ellos con una orientación feminista o ecologista, pero en todos los casos incorporando la reflexión y el análisis crítico. Algunos recursos han sido: cuento "El sueño de Álvaro" dirigido a la sensibilización hacia alumnado con discapacidad física, juego didáctico "Creciendo en Valores" o el teatro "Crecer sin Gluten", en el que se da a conocer de forma teatral las desigualdades del alumnado que tiene esta intolerancia. 
A propósito de la competencia CM2.11 el alumnado ha indagado y descrito las actuaciones de relación entre escuela y comunidad desarrolladas por distintos colectivos en la ciudad. Ejemplos de ello son MZC, con un conjunto de talleres coeducativos en las escuelas, Paz con Dignidad, a través de talleres de consumo responsable o el impacto de las multinacionales en nuestras vidas, o Plataforma contra la Violencia de Género y su trabajo educativo en los colegios.

Con respecto al profesorado participante en la innovación docente, el proyecto ha permitido:

Mejorar la coordinación docente en todos los grupos (4 grupos de Primaria y 3 de Infantil), coordinando los contenidos, actividades prácticas, calendario y criterios de evaluación. Para ello se ha hecho uso de distintas herramientas tecnológicas: plataforma ENOA donde se han compartido textos teóricos que han sido material de clase, documentos complementarios útiles para la preparación de la asignatura y otros materiales visuales, tales como cortos o fragmentos de películas, de importante valor didáctico. Otra herramienta utilizada ha sido Google Drive, donde se han ido compartiendo las actas de las reuniones de coordinación que se han mantenido, así como la transcripción literal de los grupos focales llevados a cabo como instrumentos de investigación y evaluación de la innovación.

- Fomentar la capacidad reflexiva del profesorado universitario, potenciada sobre todo a través de la realización del diario de clase. A través de este instrumento, las profesoras participantes han plasmado de forma diaria/semanal las potencialidades del día a día, los aspectos a mejorar, las reacciones del alumnado ante la propuesta de actividades en el aula o la presencia y participación de los distintos grupos.

Potenciar el diálogo y la colaboración entre las profesoras mediante la realización de grupos focales al comienzo y final de la asignatura. En ellos se han expuesto las distintas preocupaciones, temores y también las satisfacciones. Compartir esta información ha permitido aprovechar los saberes y experiencias como grupo docente y sentirse apoyadas.

Con respecto al contacto universidad-organizaciones sociales de la ciudad, el proyecto ba posibilitado:

Realización de talleres dentro del aula por parte de organizaciones sociales de la ciudad con las que el alumnado trabaja durante el desarrollo de la asignatura. Además de la iniciativa de las profesoras, el propio alumnado también tiene la oportunidad de invitar a colectivos para que asistieran a las aulas, lo que fomenta su autonomía y sentido de la responsabilidad.

Conocimiento divergente y contrastado a través de distintos puntos de vista. El hecho de recibir a distintas organizaciones y de distintas orientaciones (de carácter religioso, laico, feminista, ecologista...) posibilita en el alumnado el contraste de argumentaciones y el conocimiento de lecturas distintas de un mismo objetivo (por ejemplo con respecto a la intervención educativa en barrios de escasos recursos económicos en la ciudad).

Colaboración activa por parte del alumnado en redes de materiales educativos creadas por organizaciones sociales. En particular se oferta la posibilidad de compartir materiales didácticos creados por los y las estudiantes con perspectiva de género en la red de intercambio del proyecto "Con voz propia, III" de Mujeres en Zona de Conflicto en el que participan profesorado y educadores de toda Andalucía. En particular se mantienen reuniones con: Mujeres en Zona de Conflicto, Paz con Dignidad, Plataforma de Mujeres Poniente Sur, Entre Culturas, Madre Coraje y participación en la asamblea Plataforma Córdoba Solidaria.

\section{UTILIDAD}

Este proyecto ha sido de utilidad para el alumnado matriculado en la asignatura pues ha contribuido a que adquieran las competencias previstas, a que sus aprendizajes sean más sólidos y estén enmarcados en el contexto social y cultural de la ciudad. Igualmente, es de utilidad para el profesorado que imparte docencia en distintos grupos porque ha permitido la coordinación y diálogo constante y fluido. De forma individual, el proceso de elaboración del diario de clase también ha posibilitado la toma de decisiones de forma reflexiva permitiéndole tener un registro histórico de los avances y dificultades.

Además, ha servido a las organizaciones colaboradoras de la ciudad implicadas porque se han establecido nuevos canales de comunicación y colaboración activa con la Universidad, dando a conocer sus objetivos y actividades en el colectivo universitario. Por su parte, su participación activa mediante el desarrollo de talleres en las aulas permite incorporar nuevas voces y metodologías alternativas.

\section{CONCLUSIONES}

Destacamos como fortaleza de nuestro proyecto el diseño y puesta en marcha de actuaciones educativas en las que el aprendizaje queda reforzado a partir de lo vivencial y experiencias socio-afectivas. Para ello nuestra apuesta es hacer uso de metodologías participativas con colectivos sociales ajenos a la institución universitaria. Avanzamos en la tarea pendiente y la necesaria reflexión conjunta y por separado sobre la manera en que la Universidad y las organizaciones no formales puedan establecer puentes de colaboración que promuevan una mirada "transdisciplinar de la sociedad local y global, orientada hacia el bien común" capaz de generar un "trabajo horizontal con el tercer sector para la solución de problemas concretos" (Andrés, 2012). 
La variedad de técnicas y recursos empleados y el establecimiento de una comunicación horizontal que favorezca al grupo de estudiantes la adaptabilidad al entorno social, cultural y económico son aspectos a mantener en futuras actuaciones. Con este motivo, nos proponemos afianzar los lazos surgidos con los distintos colectivos, dar entidad al trabajo realizado por el alumnado, a través de cartas de presentación de la asignatura, estrategias de fortalecimiento y compromiso por parte de las docentes con los colectivos sociales.

Los procesos de coordinación constante y los deseos mantenidos de alcanzar un "espacio social democráticamente gobernado por el pensamiento, la crítica, la provisión y realización del bien común de la educación" (Escudero, 2006) suponen un avance en la meta de desarrollar estrategias metodológicas de acciónreflexión-acción que repercutan en la calidad docente y en la mejora del proceso de enseñanza y aprendizaje.

\section{AgradeCIMIENTOS}

Agradecemos el trabajo realizado por el alumnado colaborador de los Grados de Infantil y Primaria en el Departamento de Educación con su contribución en la codificación y transcripción de cuestionarios y grupos focales: Lorena Vallejo, Juan Pozuelo, Cristina Lázaro, Tatiana Recio, Sara Pedrajas, Nerea Gómez y Victoria Jurado.

Agradecemos la acogida y colaboración mantenida con las organizaciones sociales de la ciudad de Córdoba. La disponibilidad de la Facultad de Ciencias de la Educación en el desarrollo de las distintas actuaciones. 6006.

Proyecto financiado por el Plan de Innovación Docente de la Universidad de Córdoba (España).ref. 2015-2- 


\section{BIBLIOGRAFÍA}

Andrés, L. (2012). Universidad y compromiso social, una experiencia transformadora. En G. Celorio y A. López de Munain (Coord.), La educación para el desarrollo en la universidad. Reflexiones en torno a una práctica transformadora (pp. 14-22). Bilbao: Hegoa

Atkinson, D. (2011). Art, equality and learning. Pedagogies. Against the state. Rotterdam: Sense Publishers.

Bain, K. (2007). Lo que hacen los mejores profesores universitarios. Valencia: Universidad de Valencia.

Brubaker, N. D. 2012. Negotiating authority through cultivating a classroom community of inquiry. Teaching and Teacher Education 28, 240-250. Doi:10.1016/j.tate.2011.10.002.

Castells, M. (2012). Redes de indignación y esperanza. Madrid: Alianza editorial.

Conferencia de Rectores de las Universidades Españolas (CRUE) (2015). Institucionalización del aprendizajeservicio como estrategia docente dentro del marco de la responsabilidad social universitaria para la promoción de la sostenibilidad en la universidad. Recuperado de: http://www.crue.org/Documentos $\% 20$ compartidos/Recomendaciones $\% 20 \mathrm{y} \% 20$ criterios $\% 20$ tecnicos $/ 2 . \% 2$ OAPROBADA\%20INSTITUCIONALIZACION\%20ApS.pdf

Escudero Muñoz, J. M. (2006). La construcción de un currículo democrático y la cultura de colaboración del profesorado. Participación Educativa, 3, 12-17.

Ferguson, K., Macqueen, S. y Reynolds, R. (2014). Pre-service teacher perspectives on the importance of global education: world and classroom views. Teachers and Teaching: theory and practice, 20(4), 470-482.

Flecha, R., Racionero, S., Tintoré, M. y Arbós, A. (2014). Actuaciones de éxito en la universidad. Hacia la excelencia tomando las mejores universidades como modelo. Multidisciplinary Journal of Educational Research, 4(2), 131-150. Doi: 10.4471/remie.2014.08

Kilgo, C. A., Sheets, J. K., y Pascarella, E. T. (2015). The link between high-impact practices and student learning: some longitudinal evidence. Higher Education, 69, 509-525.

Kuh, G. D. (2008). High-impact educational practices: What they are, who has access to them, and why they matter. Washington, DC: Association of American Colleges and Universities.

Ministerio de Educación (2010). Estrategia Universidad 2015. Recuperado de: http://www.educacion.gob.es/eu2015/la-eu2015.html

Pujolàs, P. (2007). Estrategias metodológicas en la enseñanza universitaria: aprendizaje cooperativo. Disponible en http://www.unex.es/unex/servicios/sofd/archivos/ficheros/formacion/Aprendizaje Cooperativo.pps

Pujolàs, P. (2008). El aprendizaje cooperativo. Barcelona: Graó

Reynolds, R., Bradbery, D., Brown, J., Carroll, K., Donnelly, D., Ferguson-Patrick, K y Macqueen, S. (Eds.). (2015). Contesting and constructing international perspectives in global education. Rotterdam: Sense Publishers.

Wineburg, M. S. (2006). Evidence in teacher preparation: Establishing a framework for accountability. Journal of Teacher Education, 57(1), 51-64. Doi: 10.1177/0022487105284475 